\title{
Bemerkungen über die Rosinen des Handels
}

r $\mathbf{n}$

Professor Dierbach.

\section{Vor $\mathbf{r}$ o $\mathbf{t}$}

Zu den verbreitetsten Droguen, welohe das südliohste Europa und die Levame liefern, gehören die grofson und Kloinen Rosinen; sie sind in den Officinen wie in den IIitchen zu finden, und wir lernen sie schon als cin beliebtes Naschwerk im Knabenalter kennen. Sie gehïren also zu den gemeinsten Dingen, und dies ist ganz offenbar der Grund, warum sie in den Lohrbüchern der Pharmalingnosie, der medioinisohen Botanik oder der pharmaceutischen WaarenLuado so kurz abgeferligt worden; auch die Lehrbücher Jer Materia mediea gönnen ibnen meistens nut ein paar Zeilen, oder erwihnen sie gar nicht, obgleich der Valer der Arzneihunde, Hippocrates selbst, sie ungemein of in seinen Schriften nennt and von ibrem Gebrauche redet. aber die gemeinsten Dinge siod eben nicht immer die in wissenschaf licher Hinsicht am genauesten untersuchten, und so scheint es auch mit den Rosiuea zu seyn, über die ich hier einige Bemerkungen mittheilen will, wenn auch nur um die Autmerksamkeit der Aerzte und Pharmaceutea auf diesen Gegenstand zu denken, da noch so manches in nalurhistorischer Hiasicht darüber sufauklären bleibt.

\section{1.}

Elymologische Notizen.

Die Rosinen sind nichts anderes als die getronkneten 'Früchte des Weinstocts, Vitis rinite: a L.; bei den Griechen hiefsen dio grölseren otaçı, die kleineren nzaфıdıu, teides abstammend ron otapudh die Weinrebe. Die Römer

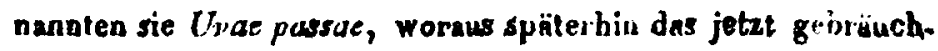


liche Passulae majores et minores gebildet worden seyn mag. Den Ansdruck passa und passula leiten mehrere Pharmakolo. gen, auf eine Stelle des Plinius sich stützend, - von patientia ab; so sagt der bekante Scbizius: Urae passae nomen suum inde acquisiverunt, quod solis calorem passae sunt. In solc enim siccantur u. 3. w. Einfacher ist es jedoch wohl, den Ausdruck vor pandere, trocknen, sbzuleiten, so dafs also ura passa nichts anderes heifst und heirsen kann, als eine getrocknele Weinbeerc, in welchem Sinne selbst scbon rörnische Schriftsteller den Ausdruck gebrauchten.

Die Weinheeren selbst hiefsen Acini und die dorin enthaltenen $S_{u m e n}$, die ehodem auch officineli woren, Vinaces, bei den Griechen Gigarta. Der deutscne Name Rosinen kommt oflenbar von dem französischen raisin, und dieser ist aeinerseits aus dem lateinischen Worte racemus entstanden, welches den eigenthümlichen Blumen - and $F_{1}$ uchtstand dec Vitis vinifera andeutet

Die getrochrieten Rosinen pflegen die Meisten, je nach. dem sie in der Sonne getrocknet wurden und einen süfsen Geschmack haben, Zibeben (oder Cibeben) oder aber wenn sie auf Oefen gedörrt wurden und süuerlich schmecken, Corinthen zu nennen. Andere verstehea unter Corinthen blos die kleineren kernlosen Rosinen mit dunbelbläulichen Beeren. $\infty$ ) Noch Andere wollen nur die grofsen Rosinen Zibeben genannt wissen, wobei zu erinnern, dafs dieser Ausdrack wesentlich nicht verschieden ist von Uva passa der Aömer, indem das urabische Zibib genau das sagt, was passa der Lateinev.****)

Nicht selten findet man auch den Ausdruck Passulae che. seminae bei den Pharmakologen, und zwar offenbar öfters

*) Quin et a patientia nomen aciois datur passie Hiat nat L. XIV $C_{2}$.

*-) De alimentorum facultatilus pag. 330.

-**) Zenker Waarenkunue Bd. I pag. 24.

\$*; Camerar. horlus medicus et philosophieus pag. 18 . 
mifsverstanden; es stammt aber auch dieses Wort aus dem Arabischen, und Kesmes oder Chesmes bedeutet nichts anderes als hernlos, wenn man also sagt passulae cheseminae, so ist dies eben so viel als passulae sine vinaceis oder apyrenae, was insbesondere von den bleinen Rosinen gilt.

9. 2.

Zubereitung der Rosinen.

Wenn wir gleich in Deatschland, zumal in den südlicheren und wärmeren Provinzen den Weinstock in Menge zieben und aus seines Trauben einen schmackbaften Wein zo bereiten wissen, so konnen wir doch keine Rosinen zubereiten, die denen gleich zu achten wären, die aus Griechenland, Kleinasien u. 2 w. kommen. Deutschlanda Klima ist lange nicht milde genug, um die zärtlicheren TraubenVarietäten sur Reife zu bringen, und jene Menge von zulkerartigen süfsen Theilen zu erzeugen, welche wir in den Hosinen des Handels zu finden gewohnt sind.

Die Zubereitung der 'Traubenbeeren zu Rosinen besteht wesentlich nur darin, dafs ersteren durch Hülte der Wärme die Feuchtigheit entzogen wird, damit sie nicht durch diese in Verderbnifs äbergehen. Es ist nber ganz und gar nicht gleichgültug, auf welche Weise dabei verfahren wird, weshalb die einzelnen Methoden lurz zu berübren sind.

1. Durch Trocknen in der Sonne. Nur die so zubereiteten Bosinen wollen einige Pharmatulogen Passulae genannt wissen. Diese Methode ist besonders bei den kleinen schwarzen Rosinen die gebrauchlichste. Die Traubenbeeren werden abgeplückt und in eigenen Behaltera der Luft and Sonne so lange ausgesetzt, bis sie die gehörige Beschaffenheit erlangt haben; sie fallen dabei bedeutend zusammen und hängon so fest aneinander, dafs man die Massen nur mittelst eigener Werkzenge roneinander bringen kann; aus jenen 
Bohätern werden sie in Pässer gefullt, mit Füfsen eingetreton und mit etwas Meerwasser besprengt. ")

Im südlichen Frankreich trocknet man die grofsen Bosinen ebenfalls in der Sonne auf Hürden, hat aber dort die Gewohnheit, die Trauben vorber sammt den Körnern in eine Sodalauge einzutauchen. ${ }^{* *}$ )

2. Durch Trocknen in geheizlen Oefen. Dies scheint in warmen Ländern kaum nöthig zu seyn, wo die Sonnen wärme und der heitere Himmel die erste Methode so sehr begünstigt. Wahrscheinlich trocknet man die Rosinen nur dann in Oefen, wenn nasse und regnerische Jahrgänge dies erheiuchen, wo dann auch der Zuckergehalt geringer zu seyn pllogt, so wird es leicht begreiflich, warum die so behandelten Rosinen sauer sind und auch nur zur Eosigbereiturg benatzt za werden pllegen.

3. Durch Trocknen im Rauche der Sihmiede-Essen. Dies ist eine der ältesten Methoden, und wird bereits ron Ca to angefuhrt, die Rosinen sollen dadurch ganz vorzüglich gut werden, aucb würden diese besonders zam medicinischen Gebrauche verwendet, und namentlich von Caelius Aurelianus empfohlen: sie dienten anter andern bei dem Husten der Schwindsüchtigen und erinnern so an das hreosot des Herrn Reichenbach zu Blansko in Mäbren.

Die Traubeasorte, welche die alten Römer rorzugsweise zur Rosinenbereitung benutzten, hiefs Vilis aminaea und scheint entweder ein Gutedel oder ein Mushateller zu seyn. ***)

Spie lma an Mater. med. pag. 97.

*) Dictionnaire des Drogues. T. IV. p. 368.

*) Man vergleiche Sickl or Geschichte des Obstes der Alteu pag. 28 . 
4 Durch Trocknen am Stocke selbst. Diese Methode ist besonders in Spanien gebräuchlich und komme scbon bei den ätestea Schriftstellern über Landwirthachaft ror; man schnei. det zu dem Ende die Fruchtstiele der zu Rosineo bestimmten Trauben zur Hälfte durch, am so den Andrang der Safte su dea Früchten abzuleiten and so das Austrocknea za bofördern. A of diese Art läfst man sie so lange an Stocke hängen, bis sie durch dep Einflufs der Sonne und aus Mangel an Nabrung zareichend eingeschrumpt siod, worauf mon sie abschneidet nnd sogleich verpackt. Dies sind die cogenannten Passerillas de Sal, die besonders aus Granada bommen. ")

5. Zubereitung der Malaga Rosinen. Nach Edv. Antill wird dabei folgendermafsen verfahren. Man bereitet eino Lauge aus Weinreben-Asche, ungefäbr halb so stark als gewöhnliche Seifensiederlauge, bringt sie ins Kochen and setat zu vier Maafs Flüssigheit (Congius) eine Hand voll Hochsalz and ein Pfund Baumöl oder anderthalb Pfund Buttex zu. In diese noch heifse Lange werden die Trauben bündelweise eingetancht, so dafs alle Theile zureichend durchdrungen werden. Nachdem man sie an dem Geschirro heransgezogen, breitet man sie auf Struhmatten aus, die unter ein bewegliches Dacb gestellt werden, das am Tage bei heiterem Wetter enternt wird. Die Traubenbündel liegen da einzeln in Meihen und müssen öfters bis zur gehörigen Trockne umgewendet werden, worauf man sio in wohl zo verbittendo und verklebte Tonnen oder Topfe bringt. *) Dies sind die Passcrillas de Lavia oder Loxia, die besonders häufg in die nördlichen europäischen Länder verfübrt werden.

-) Mar oebe Dale Pharmacologia pag. 626

- Eergius Maleria medica pag. 162. 
Uebrigem haben die Spanier dieses Verfabren offenbar von den Römern erlernt, indem bereits Columell. dasselbo fast auf ganz gleiche Weise anfiuhrt. ")

Poltrosinen oder Topfrosinen sind nach Martius eirgetrochnete spanische Weintrauben, die man in Töpfen mit Ralk amschüttet za aus bringl, die aber doch wohl zu den Seltenheiten gehören. Griechen und Römer kannten mancherlei Methoden, die Trauben geraume Zeit so aufzubewahrea, dafs sie ihre äufsere Form und Saftigheit behielten, was nicht weiter hierher gebört. **)

\section{3.}

\section{Uebersicht der bekanatesten Rosinensorten.}

Wir haben schon oben gesehen dafs gute Rosinen nur in Iändern, deren Klima ein sehr mildes ist, erhalten werden h6anea; besonders scheinen die Gegenden, welche das mittelländische Meer bespült, dazu am geeignetsten zu sejn; and nur allenfalls in einigen Distriluten ron Ungarn noch Lönnten Rosinen erbalten werden, ron wober doch dergleichen wenigstens heutzutage beine in des Handel homren, obgleich noch $\mathbf{C}$ artheuser in seined bekannten Scbriften von ungrischen Rosinen redet. Es lassen sich aber uaterscheiden :

- Französische Rosinen: Passulae valgares vel gallieae, auch Kisten-Rosinen, Raisins de Caisse genannt. - Sie aind gelb and sehr süls. anch werden davon viele nach Afrika rerschickt, wo sie, zumal auf dez Carawanenzügen durch die Wüsten, verzohrt werden. Sie gehören zu den Früchtea, die man nach Guille min etras seltan les quatre mendians nenne.

-) De re rustica XII Cap. 26.

*) Man vergleicbe Coiumella Lib. XII Cap 43 Baplies Porta de Villis pag. 5,8 . 
Dia französiachen Rosinen Lommen besonders ans Languedoc und der Provence (sie beifsen auch Jubis oder Robis, Piccarden, Muscatrosinen), von Toulon, Aubagne, Pezena, besonders aus Marseille, Pussulae massiliaticue; zn den Zeiten des Tabernaemontanus hielt man diese fur die besten, auch $Z$ or $n$ nennt sie eine ganz vorzügliche Sorte, die den gewöhnlichen oder Korb-Rosinen vorzuzieben sey; sie haben nach ihm eine schwarzblaue Farbe.

2. Italische Rosinen. Nur in den südlicben Theilen dieses Landes, zumal in Calabrien, Sicilien ond auf den lipari. schen Inseln scheint man sich damit zu befassen, denn die Triester Rosinen, deren Martius gedenht, stammen Laum aus der dortigen Gegend. Die aus Calabrien hommenden sind auf Fäden gereihet und werden vielleicht noch, wio einst im Alterthume, in dem Raucbe der Schmiede - Essen getrocknet; sie sind grofs, sebr süfs, blaulich oder grau und haben sonst mit den spanischen grofse Aehnlichleit. Ob die sut Bindfaden gereiheten und zur Esaigbereitung dienenden Belvederrosinen, wovon Martius redet, aus Itlien oder aus andern Gegenden kommen, konnte ich nicht erfahren. Sonst brachte man aus Italien viele Hosinen in Iörbe gepacht (Korb-Rosinen), die aber nicht sehr geachtet waren. Auch die lipurischen Corinthen werden blos zum Färben benutzt.

Die Hömer scheinen zu den Speisen sich blos der grofsen Rosinen bedient zu haben, indem Apicius in seinem Werthe über die Kochlunst immer anmerkt, daf man die Kerne herausnehmen müsse.

3. Griechische Rosinen. Im Alterthume scheint man vorzugoweise nur grofse Rosinen zubereitet za haben, wie schon aus dem Namen der Traubon (Boumasti, Ochsenauge) hervorgeht, die sie daza verwendeten. *)

$\rightarrow$ Der Spanier Clemonte de Rola hat mebrere Trauben- 
Die jetzt bei uns vorkommenden Sultania.Rosinen dürften auch griechischen Uraprunges seyn; sie sind gelb, bodeutend groffer als die gemeinen blauen Corinthen, aber ach riel tleiner als die gewöhnlichen grofsen Rosinen, ron. Gestalt meistens ganz rund und ohne Kerne; auch stehen sie hơher im Preise als die übrigen hänflichen Rosinensorten.

Der Rosinen aus der Insel Somos gedentit Marti us; besonders gebören aber die allbekannten tleinen Hosinen oder Cortmithen hierher, die lange aus der Gegend von Corinth Hamen und davon ihren Namen erhielten, jetzt aber von den Inseln des jonischen Meeres, Ithaea, Cephalonia, Zacynthus, aber überdem noch aus Morea, Vortiza, Patras, Missolunghi u. s. w. Lommen. Es sollen jährlich an 17 Millionen Plunde verfuhrt werden, wovon sieben auf die Insel Zante kommen, im Werthe ron drei Millionen Franken. Nach Spielmann werden jährlich an 100,00o Centner verführt. - In den jüngsten Zeiten machte man den Versuch, die Corinthentrauben nach Corsika zu verplanzen, um diesen einträglichen Handeiszweig den Franzosen zuzawenden. Es gibt nebat den gemeinen schwarzen auch blaue, rothe und luhfarbige Corinthen.

Nach Marti us werden die Corinthen-Trauben dann gesammelt, wenn ibre rothe Farbe in das Purpurrothe geht; man legt sie in Körben auf eine abhängige Tonne, um das Ablaufen des freiwillig ausliefsenden Saftes 20 begünstigen und wendet sie öfters; nach dem Trooknen entfernt man die Hämme und bringt die Rosinen in Fässer.

4. Spanische Rosinen. Passulae hispanicae. So wie Spanien besonders reichlich sufse und geistreiche Weine liefert,

sorten mit diesem Namen beseichnet; im Spanischen heifsen sie Tetas de Vaea und zeichnen sich durch die Gröfse der Beeren aus; es geh6ren dahin Vitis vinifera sulcata, lougisaima, macrobotrys u. s. $w$. 
eben so reichlich und vortrefflich sind die Mosinen, die aus dieser fruchtbaren Halbinsel kommen. Besonders berühmt and geschritut oind die Malaga-Mosinen, auch Moskateller. Hosinen genannt; sie sind in Deutschland sehr verbreitet, wie men denn namentlich in den Hamburger Droguen Berichten meistens nur diese angeführt findet. Elne aus diesen erlesene Sorte sind die Kloster-Rosinen, wovon die tchön. sten und besten in den Weiabergen bei Velex Malaga erzielt werden. Granada liefert zwar sehr viele Bosinen, die jedoch weniger geachtet sint als die vorigen. Aus Spanien stammen forner die bläulichen. Aeischigen Pickrosinen, Pickziber ben oder lange fosinen, die sich durch ihren böchat süfsen lieblichen Geschmack ouszeichnen; eino indere lichtgraue Sorte ist zwar grofser, aber weniger schmachhaft and wird darum anch minder geschätzt als die vorigen,

Endlich sind noch die Allcant-Rosinen zu erwihnen, die jedocb im Grunde nichls anderes sind, als Rosinen aus der spaniachen Provinz Valencia.

Abbild. Zienker Waarenkunde Taf, III fig. 3.

5. Levantische Rosinen. Von den ältesten Zeiten an bis auf den heutigen Tag wurden Rosinen aus mehreren Provinzen der Levante, inshesondere aus Klein-Asien und Syrien nach Furopa in den Handel gebracht. Nach Galen kameo ehemals dergleichen aus Cilicien, Galatien und Pamphylien, auch ist es. nicht unwahrscheinlich, dafo die Rosinentrauben ursprünglich aus dem Orient nach Corinth und von da nach den jonischen Inseln rerpflanzt wurden; aus Cilicion kamen vorzugsweise kleine Rosinen, aus Pamphylien gröfrere schwarze, und so wird es verständlich, warum die Corinthen von einigen Pharmakologen Passulas Ciliciae genasnt werden. Galen hannte übrigens schon Passulae subflavae. nigrae, dulces, subausterae und einige andere.

Heulzutage erhäll man ans Asien noch folgende Sorten: 
a. Rosinen aus Dămascus; Passulae damascenae. Si werden in Schachteln verschicht, sind fast einen Zoll lang platt gedrücht, bisweilen auch rund, branngelb, von seh süfsem zacherartigen Geschmacke und angenehmem Mushat geruche.

6. Rosinen aus Smyrna: sie gleichen den vorigen sehr werden aber in Fässern rerpackt nach Europa.geschicht; e. gibt davon a Spielarten, nämlich rothe (Carabuno) u. schwarze Abbild. Zenker Wasenkunde Taf. IV fig. 1. 2.

\section{4 .}

Uebersicht einiger zu Rosinen tauglichen Varieläten des Weinstocks.

Kaum ron irgend einem andern Gewächse keant man so xahlreiche Formen und Spielarten als von der Weinrebe, dic in Hinsicht der Grörse und Stärke des Stammes, der Beschaffenheit der Blatter und zumal der Früchte die mannichlaltigsten Verschiedenheiten zeigen, und was hier besouders in Betracht koromt, auch in Rücksicht ihrer Vegetationsart und Reifzeit merklich von einander abweichen. daher es ein Umstand von der grölsten Wichtigkeit für den Weinbau ist, in jeder Gegend genau die ibrem Boden und ondern Verhältnissen angemessensten Sorten mit kluger Umsicht auszunählen. Die Rosinentrauben gedeihen nur im wärmeren Theile von Europa; man wird sie daher in Deutsch. lands Weinbergen vergeblich suchen, und höchstens nur in Gärten findet sich hie und da eine oder die andere von einem Liebhaber der Rebenucht als Seltenheit cultivirt.

Man kann sie folgendermafsen anordnen:

A. Corinthentraubea mit kleinen sameolosen Früchten.

a. Vilis corinthiara Risso. *) Acino minaco subsphacrico virescente pellucido, succo dulciori.

") Histoire natur. des product. de l'Europe merid. Vol. II p. 188. 
V. corinthiaca. Folits ovatis subtrilobis subtus villosis, ura mediocri subllaccida, acinis ochrolencis pruina alba obductis, epicarpio tenui operculato, sarcocarpio succulento saceharato. Linnaea. Vol. 3 p. 147.

Uva passa bianca. Onomatoloģia bot. V. 9 p. 507 Nr. 145.

Diese Varietät der Vitis vinifera ist offenbar die Mutterpllanze der Sultania-Rosinen, and diese sind offerubar die Pesulae ciliciae des $\mathrm{Gal}$ en; er sagt rergleichend mit den grofsen Rosinen: Aliae quaedam sunt his contrariae colidae et crassee, quae nullum prorsus semen habent. Crescunt hae in Cilicia, colore sublaro a. \&. $w, *)$

Es gibt davon auch eine schwarze Spielart. Uva pasca nera der Onomatologia botanica Nro. 146.

b. Vitis apgrena Risso (loco citato). Acino minino absphserico pellucido, succo dulci, semine saepe carente.

Der rorigen nabe verwandt, aber durch die noch auffallendere Kleinheit alle Theile deutlich verschieden.

c. Vitis minuta Rissw (pag. 210). Acino minimo elliptico, niguo-violaceo, succo duici, semine carente.

Corintheutrauben, Weisbeerleiv. Orromatciogin botan. low cit. Nro. 36.

V. passula: acinis minirnis nigerrimis dulcisoimis, plerumpe aspermis. Presi. Flor. Sical. p 201.

V. acinis onnium minimis, sole siccari statutis. Cup. hort. cathol. 234 .

Tuccarineddu, Niuriduzzu, chi caicalu, ctoe impmesito, vien chiamato Passuiana di iu nortru Regnn. Sical.

Abbiad Zenker Warenkunde Bd. I Tab. III fig.

Plenk plant. medicin. to 145.

Dässeidorf. Sammlung 11. Lief. Nro. 5.

Dies ist die Mus erplans: der geucinen wituea schrarzen Ausinen oder Corintaveo.

†) De atimusturun facuitatibus :ib. 2 Cajs. 10. 
B. Hosinentrauben mit grofren langen samenlosen Früchten.

a. T'itis asperma: acinis magnis duris flavis suavibus sub. moschatis aspermis. Presl. flor. Sicul. Vol. I p. 203.

V. oblongo fructu majori rarulo calloso flaro suavi gustu ac nonnihil moschum sapiente Cup. hort. cathol. 233.

Zibibu Sicul.

Diese eigenthümliche Posinen - Art scheint bei uns nicht häa. fig rorzulommen.

C. Rosinentrauben mit grofsen runcien samesbaltigen Früchtex.

V'itis apiana Plinii. Die Muskatellertraube, Zibebentraube. Grolse Rosinentraube.

Zenker Waarenkunde Bd. I Taf: II optime !

Mehrere besondere Spielarten, welche grolse rande Rosinen liefern, gehören noch hierber.

a. Vitis apiana Risso (rag. 226) acino medio sphaerico nigro- violaceo, succo saccharato moschato.

V. apiana. Clemente de Roxas (Moscatelles) mit zahlreichen in Spanien häufig cultivirten Spielarten : generosa, moschata, oborats, Isidori, pseudo - apiana, Elisabeth a. s. w. Roemer et Schultes System. Vegetabil. T. V p. $30 q$

V. apiana: Foliis $3-5$ lobis planis subtus pilosulis, uva magna densa, acinis ochrolencis punctatis pruinosis, epicarpio denso, sarcocarpio duriusculo, sapare peculiari moschato rel hibis nigri baccas aemulante Linnaea I. c. p. 151. Variat acinis rabris, coeraleis et nigris

Dies ist die Mutterptlanze der so beliebten grofsen spaniachen oder Muskateller - Rosinen.

b. Vitis massiliensis Risso (p. 228). Acino magno sphaerico, rubro violeceo- nigro, succo dulci eaccharato.

Diese mit ihren verwandten Formen liefert die von unsern Vorfahren 80 geschätzten Marseiller Rosinen. *) Dahin

-) Man rerglejche Cbomel A brégé do l'hintoire des plantes usuelles. Paris 1725. Vol. I pag. 110. 
a. Rosinen ous Damasus: Passulae damascenae. Sie werden in Schachtelo verschicht, sind Mst einen Zoll lang, platt gedrückt, bisweilen auch rund, braungelb, von sehr süfsem zacherartigen Geschmacke und angenehmem Mushatgeruche.

6. Rosinen aus Smyrna: sio gleichen den vorigen sebr, werden aber in Füssern rerpackt nach Europa geschickt; es gibt davon 2 Spielarten, nämlicb rothe (Carabuno) u. schwarze. Abbild. Zenker Waarenkunde Taf. IV fig. I. s.

6. 4 .

Uebersicht einiger zu Rosinen tauglichen Varietäten des Weinstocks.

Kaum von irgend einem andern Gewächse kennt man so zablreiche Formen und Spielarten als von der Weiarebe, die in Hinsicht der Grörse und Stärke des Stammes, der Beschaffenheit der Blätter und zumal der Früchte die mannichfaltigsten Verschiedenheiten zeigen, und was hier besonders in Betracbt koromt, auch in Rücksicht ihrer Vegetationsart und Reifzeit merklich ron einander abweichen, daher es ein Unstand von der gröfsten Wichtigheit für den Weinbau ist, in jeder Gegend genav die ihrem Boden und andern Verhöltnissen angemessensten Sorten mit hluger Umsicht auszuwählen. Die Hosinentrauben gedeihen nur im wärmeren Theile von Europa; man wird sie daher in Deutachlands Weinbergen vergeblich suchen, und böchstens nur in Gärten findet sich hie und da eine oder die andere ron einem Liebhaber der Rebenzucht als Seltenheit cultivirt.

Man kann sie folgendermalsen anordnen:

A. Corinthentraubea unit kleinen samealosen Früchten.

a. Vitis corinthiara Risso. *) Acino minaco subspbacrico virescente pellucido, succo dulciori.

-) Histoire natur. des product. de l'Europe merid. Vol. II p. 186. 
Linneen 1. c. p. 251 . Onomatolegia botanica Nro. 41 (variet. sphaerica ).

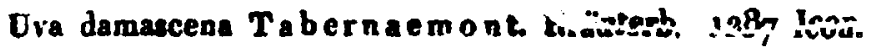

Zenther Waaronkande Yol. I Taf. 3 fig. D.

c. Vitis maroccuna Risso ( 0.335 ), Acino maximo cordiformi, rubro- violaceo saceo dulai

P. nfricana. Lisnaeu 1. c. p.52.

d. Ia is Salernitana Risso (p. 131 ). Acino medio ovato nigr 0 - violaceo, succo dulciseimo.

e. I'itis alexandrina: foliis quinquelotia subg'abris, apa magne flaceida romosa, acinis insequalibue riridı-fleris, praina slba obductis, epicarpin denso, sarcocarpio sapidiesimo aromatico.

Muscateller von Alexandria, Muscat à gros grains, Hub cat long, Pála murgué, fungoon da cog. Vitis peroz̧olacar Wit dreicchigen unten b!afszrünen Bläktern, grofsen, sehr langen, weicheerigen Trauben, deren grofse, Aleischige and gelbliche Beeren einen s:arken Musbatelleryeschnack und eine Form fast wie Plauuen haben, zu ibrer Zeiriga.g eine sehr neifse Mittagsounne erfordera und spät veif wer den. Onomatolog. bot. p. 594. Nr. 112.

Vitis pergulana, acinis pranoram magnitudine et forme. C. Bauh. Pin. 298. Tilli Catalogas piantarum horti Pisani pag. 173.

Dies sind die Trabbencorten, ron welchen rorzagsweive jene lhasines erbalten werden, ron denen oiven urtier dem Namen der levantiscinen die kede war. 